\title{
The novel ATP-sensitive potassium channel opener iptakalim prevents insulin resistance associated with hypertension via restoring endothelial function
}

\author{
Yu WANG ${ }^{1, \#}$, Fu-hu ZENG ${ }^{2, \#}$, Chao-liang LONG ${ }^{1, \#}$, Zhi-yuan PAN ${ }^{1, \#}$, Wen-yu CUI ${ }^{1}$, Ru-huan WANG ${ }^{3}$, Guo-shu LIU ${ }^{2}$, \\ Hai WANG ${ }^{1,3, *}$ \\ ${ }^{1}$ Cardiovascular Drug Research Center, Institute of Health and Environmental Medicine, Academy of Military Medical Sciences, Beijing \\ 100850, China; ${ }^{2}$ Department of Cardiology, Chinese People's Liberation Army General Hospital, Beijing 100853, China; ${ }^{3}$ Thadweik \\ Academy of Medicine, Beijing 100039, China
}

Aim: To investigate the effects of iptakalim on endothelial dysfunction induced by insulin resistance (IR) and to determine whether iptakalim improved IR associated with hypertension in fructose-fed rats (FFRs) and spontaneously hypertensive rats (SHRs). Methods: Human umbilical vein endothelial cells (HUVECs) were used for in vitro study. The levels of endothelial vasoactive mediators and eNOS protein expression were determined using radioimmunoassays, ELISAs, colorimetric assays or Western blotting. SpragueDawley rats were fed with a high-fructose diet. In both FFRs and SHRs, tail-cuff method was used to measure systolic blood pressure (SBP), and hyperinsulinemic-euglycemic clamp was used to evaluate IR states.

Results: (1) Cultured HUVECs incubated with the PI3-kinase inhibitor wortmannin (50 nmol/L) and insulin (100 nmol/L) induced endothelial dysfunction characterized by significantly reduced release of NO and expression of eNOS protein, and significantly increased production of ET-1. Pretreatment with iptakalim (0.1-10 $\mu \mathrm{mol} / \mathrm{L})$ could prevent the endothelial dysfunction. (2) In FFRs, the levels of SBP, fasting plasma glucose and insulin were significantly elevated, whereas the glucose infusion rate (GIR) and insulin sensitive index (ISI) were significantly decreased, and the endothelium-dependent vascular relaxation response to ACh was impaired. These changes could be prevented by oral administration of iptakalim (1, 3, or $9 \mathrm{mg} \cdot \mathrm{kg}^{-1} \cdot \mathrm{d}^{-1}$, for 4 weeks). The imbalance between serum NO and ET-1 was also ameliorated by iptakalim. (3) In 2-4 month-old SHRs (IR was established at the age of 4 months), oral administration of iptakalim (1, 3, or $9 \mathrm{mg} \mathrm{kg}^{-1} \cdot \mathrm{d}^{-1}$, for 8 weeks) significantly ameliorated hypertension and increased the GIR to the normal level.

Conclusion: These results demonstrate that iptakalim could protect against IR-induced endothelial dysfunction, and ameliorate IR associated with hypertension, possibly via restoring the balance between NO and ET-1 signaling.

Keywords: endothelial dysfunction; insulin resistance; hypertension; ATP-sensitive potassium channel opener; iptakalim; spontaneously hypertensive rats; fructose-fed rats

Acta Pharmacologica Sinica (2011) 32: 1466-1474; doi: 10.1038/aps.2011.129; published online 7 Nov 2011

\section{Introduction}

Epidemiological evidence has demonstrated that insulin resistance (IR) and compensatory hyperinsulinemia play important roles in the onset and persistence of essential hypertension ${ }^{[1-4]}$. IR has been reported in several animal models of hypertension, including spontaneously hypertensive rats (SHRs) and fructose-fed hypertensive rats (FFRs) ${ }^{[5]}$. Humans with a genetic predisposition to hypertension tend to develop IR with hyperinsulinemia ${ }^{[6]}$. Because IR and hypertension often

\footnotetext{
\# These authors contributed equally to this work.

* To whom correspondence should be addressed.

E-mail wh9588@yahoo.com.cn

Received 2011-04-18 Accepted 2011-08-30
}

co-existed, the effects of antihypertensive drugs on IR have been highlighted. Of the first-line antihypertensive drugs, only angiotensin-converting enzyme (ACE) inhibitors and angiotensin II (Ang II) type 1 (AT1) receptor antagonists have been reported to have benefits in patients with $\mathrm{IR}^{[7]}$. However, despite intense research, studies identifying drugs able to lower blood pressure and reverse IR are still lacking.

Recent research indicates that endothelial dysfunction plays a key role in linking the pathogenesis of hypertension and IR, and the vicious circle involving hypertension, IR, and endothelial dysfunction has been documented ${ }^{[8-11]}$. Endothelial dysfunction associated with hypertension and IR is often characterized by an imbalance between NO and ET-1 signaling $^{[12]}$. Therefore, restoration of endothelial function may be 
a useful way to inhibit the development of IR in hypertensive patients.

The pharmacological approach to restoring the balance between nitric oxide (NO) and endothelin-1 (ET-1) signaling in the endothelium has been well documented ${ }^{[13]}$. A promising candidate in this field is iptakalim, a novel adenosine triphosphate (ATP)-sensitive potassium $\left(\mathrm{K}_{\mathrm{ATP}}\right)$ channel opener that has been shown to have antihypertensive effects in different animals models of hypertension and in humans ${ }^{[14,15]}$. Our laboratory has recently demonstrated that iptakalim can protect against endothelial dysfunction induced by cardiovascular risk factors, such as homocysteine, hyperglycemia, and hyperuricemia, by activating the SUR2B/Kir6.1 subtype of $K_{\text {ATP }}$ channels in endothelial cells ${ }^{[16-19]}$. Because iptakalim has highly efficacious antihypertensive and potent endothelial protective effects, we hypothesized that iptakalim treatment has beneficial effects on the IR associated with hypertension, through protecting endothelial function.

Here, we first investigated the protective effects of $\mathrm{K}_{\mathrm{ATP}}$ activation by iptakalim on endothelial dysfunction induced by insulin and wortmannin in human umbilical vein endothelial cells (HUVECs) and then explored its effects on IR associated with hypertension in fructose-fed rats (FFRs) and spontaneously hypertensive rats (SHRs). We found that iptakalim improved the IR associated with hypertension by restoring the balance between nitric oxide and endothelin-1 signaling, which was a different mechanism than that of drugs affecting the renin-angiotensin system (RAS). These investigations provide experimental evidence for a novel strategy to treat the IR associated with hypertension.

\section{Materials and methods Chemicals}

Iptakalim was synthesized by the Thadweik Academy of Medicine (Beijing, China). Rosiglitazone, insulin, wortmannin, and glibenclamide were purchased from Sigma-Aldrich (St Louis, MO, USA). Benazepril was a gift from Novartis (Beijing, China).

\section{Endothelial dysfunction induced by insulin resistance with hyperinsulinemia in vitro}

Human umbilical vein cells (HUVECs) were obtained from Cascade Biologics (Portland, OR, USA). Cells from the third to fourth passage were used for experiments. To mimic IR, wortmannin, a partial inhibitor of phosphoinositide 3-kinase (PI3-kinase), and an high concentration of insulin were added to the cells ${ }^{[20]}$. Briefly, HUVECs were preincubated with or without iptakalim $(10 \mathrm{nmol} / \mathrm{L}-100 \mu \mathrm{mol} / \mathrm{L})$ for $6 \mathrm{~h}$ and then incubated with wortmannin $(50 \mathrm{nmol} / \mathrm{L})$ and insulin $(100$ $\mathrm{nmol} / \mathrm{L})$ for $20 \mathrm{~h}$. Glibenclamide $\left(10^{-5} \mathrm{~mol} / \mathrm{L}\right)$ was added $1 \mathrm{~h}$ before iptakalim pretreatment. The medium was collected for the NO, ET-1, 6-Keto-PGF1a (namely 6-keto, a PGI 2 metabolite) and PAI-1 assays. The level of NO, including nitrite and nitrate, was measured using a commercial kit (Nanjing Jiancheng Bioengineering Institute, Nanjing, China); PAI-1 was measured by ELISA using a commercial kit (Beijing BIO-LAB Materials Institute, Beijing, China); and the other substances were measured using a commercial radioimmunoassay kit (Eastern Asia Radioimmunity Research Institute, Beijing, China) according to the manufacturer's instructions. The expression of eNOS protein was detected using Western blot analysis. The methods for cell lysis and Western blot analysis have been described previously ${ }^{[21]}$. The monoclonal anti-eNOS antibody was used at the manufacturers' suggested concentration. A secondary antibody (rat anti-rabbit IgG-horseradish peroxidase) and an enhanced chemiluminescence kit were used to visualize the immunoreactive bands. Multiple exposures of films were obtained to determine the optimal exposure time. The protein bands were scanned by a densitometer, and the relative intensities were quantified using ImageQuant software.

\section{Animals and drug treatments}

Six-week-old male Sprague-Dawley (SD) rats were provided by the Experimental Animal Center of the Beijing Institute of Pharmacology and Toxicology. Male SHRs and normotensive Wistar-Kyoto (WKY) rats of various ages were obtained from the Institute of Cardiovascular Diseases, Chinese Academy of Medical Sciences (Beijing, China). The rats were housed in groups of five, under a 12-h light/dark cycle at a temperature of $24 \pm 1{ }^{\circ} \mathrm{C}$ and relatively humidity of $56 \% \pm 10 \%$, with free access to water and a normal diet (22\% protein). All animal procedures were performed in accordance with the Declaration of the National Institutes of Health Guide and Use of Laboratory Animals (Publication 85-23, revised 1985) and approved by the local animal care and use committee.

Six-week-old male Sprague-Dawley rats were divided into 7 groups ( $n=14$ per group) and fed ad libitum one of the following diets for 8 weeks: standard chow (control rats), standard chow with iptakalim $\left(9 \mathrm{mg} \cdot \mathrm{kg}^{-1} \cdot \mathrm{d}^{-1}\right)$, a high-fructose diet (fructose-fed rats), or a high-fructose diet with iptakalim $(1,3$, or 9 $\left.\mathrm{mg} \cdot \mathrm{kg}^{-1} \cdot \mathrm{d}^{-1}\right)$ or rosiglitazone $\left(5 \mathrm{mg} \cdot \mathrm{kg}^{-1} \cdot \mathrm{d}^{-1}\right)$. During the last 4 weeks, all the medications were given daily by gavage. In a separate study, the effect of iptakalim $(3 \mathrm{mg} / \mathrm{kg}$ ) on endothelial function in the fructose-fed rats during the last 4 weeks was compared to enalapril ( $3 \mathrm{mg} / \mathrm{kg}$ ). The high-fructose diet contained $60 \%$ fructose, $13 \%$ fat, and $20 \%$ protein by calories. Body weight, systolic blood pressure (SBP), and heart rate (HR) were determined every week throughout the study. The SBP and HR were measured using a tail-cuff method in conscious rats.

Both SBP and HR were elevated, while IR was not established in the 2- and 3-month-old SHRs. IR was established in the 4-month-old SHRs. The therapeutic arrangement was carried out before (2-3 months of age) and after (4 months of age) IR was established. SHRs aged 2, 3, or 4 months were randomized into five groups ( $n=5$ rats/group) and treated daily for 8 weeks by gavage with iptakalim $\left(1,3\right.$, or $\left.9 \mathrm{mg} \cdot \mathrm{kg}^{-1} \cdot \mathrm{d}^{-1}\right)$, benazepril $\left(6 \mathrm{mg} \cdot \mathrm{kg}^{-1} \cdot \mathrm{d}^{-1}\right)$, or vehicle. Six age-matched WKY rats were given vehicle alone for 8 weeks. 


\section{Hyperinsulinemic-euglycemic clamp}

Euglycemic-hyperinsulinemia clamp analysis was performed as described previously ${ }^{[22]}$. Briefly, at the end of 8 weeks, rats were fasted for $12 \mathrm{~h}$ before the start of the experimental protocol. Then, they were anesthetized by an intraperitoneal injection of pentobarbital sodium $(50 \mathrm{mg} / \mathrm{kg})$. The left carotid artery (for blood sampling) and both femoral veins (left femoral vein for exogenous glucose infusion and right femoral vein for insulin infusion) were cannulated with silastic catheters. The catheters were flushed periodically with heparinized saline to maintain patency. At the beginning, blood samples were collected to measure basal plasma glucose concentrations, and then a constant infusion of insulin at a rate of 10 $\mathrm{mU} \cdot \mathrm{kg}^{-1} \mathrm{~min}^{-1}$ was performed. Meanwhile, a variable infusion of $10 \%$ dextrose was adjusted based on 5-min blood samples to maintain the plasma glucose at the target value. A stable plasma glucose concentration and an exogenous glucose infusion rate (GIR) were generally achieved within 70 to $90 \mathrm{~min}$, at which time 6 blood samples $(200 \mu \mathrm{L})$ were collected at 5 -min intervals to determine the GIR.

\section{Metabolic and endothelial function measurements}

Blood samples were obtained from the retro-orbital sinus from rats that were fasted overnight and euthanized with ether. Plasma glucose levels were determined with an automated biochemical analyzer (7600-DDP-ISE; Hitachi Software Engineering, Yokohama, Japan). The concentration of insulin, NO, ET-1, and Ang II levels in the plasma were measured using a commercial radioimmunoassay kit (Eastern Asia Radioimmunity Research Institute, Beijing, China) according to the manufacturer's instructions. The NO level was measured using a commercial kit (Nanjing Jiancheng Bioengineering Institute, Nanjing, China). Insulin sensitivity was assessed using the quantitative insulin sensitivity index [ISI=- $\ln$ (fasting glucose level×fasting insulin level)].

Determination of the endothelium-dependent vascular relaxation The thoracic aorta was isolated and removed from high-fructose diet rats after 4 weeks of vehicle or drug therapy. Acetylcholine-induced endothelium-dependent vascular relaxation was tested as described previously ${ }^{[23]}$.

\section{Statistical analysis}

The data are expressed as the mean $\pm S D$, and $n$ indicates the number of experiments. For multiple comparisons, the results were analyzed by factorial ANOVA using the Statistical Analysis System software (SAS8.00). A value of $P<0.05$ was considered to be statistically significant.

\section{Results}

\section{Activation of $\mathrm{K}_{\mathrm{ATP}}$ reverses endothelial dysfunction in vitro}

The experiments were carried out in cultured HUVECs to investigate the improvement in $\mathrm{K}_{\mathrm{ATP}}$ channel activation by iptakalim in vitro. Endothelial dysfunction was induced with wortmannin to block PI3-kinase-dependent signaling, and meanwhile, the cells were exposed to high concentrations of insulin $^{[20,24]}$. The release of NO (Figure 1A) and 6-keto- $F_{1 \alpha}$ (Figure 1B) decreased, and the levels of ET-1 (Figure 1C) and PAI-1 (Figure 1D) increased in the HUVECs incubated with wortmannin and insulin at concentrations of 50 and $100 \mathrm{nmol} / \mathrm{L}$, respectively, for $20 \mathrm{~h}$. Pretreatment with iptakalim at concentrations ranging from $10 \mathrm{nmol} / \mathrm{L}-10 \mu \mathrm{mol} / \mathrm{L}$ for $6 \mathrm{~h}$ increased NO release (Figure 1A) and prevented ET-1 formation (Figure 1C). Iptakalim had mild effects on $6-$ keto- $\mathrm{F}_{1 \alpha}$ release only at the high concentration of $10 \mu \mathrm{mol} / \mathrm{L}$, which was 100 times greater than the effective concentration for increasing $\mathrm{NO}$ release (Figure 1B) but had no effect on the API-1 level (Figure 1D). The effect of iptakalim on NO release was abolished by glibenclamide $(10 \mu \mathrm{mol} / \mathrm{L})$, a specific blocker of $\mathrm{K}_{\text {ATP }}$ channels (Figure 1E). In addition, eNOS protein expression in HUVECs was decreased when incubated with wortmannin and insulin, while pretreatment with iptakalim could significantly increase eNOS expression (Figure 2). These results suggested that iptakalim protects against endothelial dysfunction mainly by restoring the balance between $\mathrm{NO}$ and ET-1 via opening the SUR2B/Kir6.1 subtype of $\mathrm{K}_{\mathrm{ATP}}$ channel in the endothelium, which is consistent with a previous report from our lab ${ }^{[16]}$.

\section{Iptakalim improves IR associated with hypertension in vivo}

First, the effect of $\mathrm{K}_{\mathrm{ATP}}$ activation by iptakalim on the progression of IR associated with hypertension was investigated in SHRs. SBP, HR, and the GIR were compared between the SHRs and age-matched Wistar-Kyoto rats (WKY). SBP and GIR in the 1-month-old SHRs and age-matched WKY rats were comparable. SBP was elevated, and GIR was normal in the 2-3-month-old SHRs. SBP was elevated, the GIR was decreased, and hypertension and IR developed in the 4-6-month-old SHRs (Figure 3A). The effect of treatment with iptakalim at doses of 1,3 , or $9 \mathrm{mg} \cdot \mathrm{kg}^{-1} \cdot \mathrm{d}^{-1}$ for 8 weeks in SHRs before or after IR had developed was evaluated. SBP decreased, and GIR increased in the SHRs treated with iptakalim at 2, 3, or 4 months of age. Treatment with iptakalim improved IR associated with hypertension, whereas treatment with benazepril at a dose of $6 \mathrm{mg} \cdot \mathrm{kg}^{-1} \cdot \mathrm{d}^{-1}$ for 8 weeks decreased the SBP but did not improve the decrease in the GIR (Figure 3B).

Second, we investigated the effects of iptakalim on IR associated with hypertension in the FFRs. Food intake was significantly lower in the FFRs compared to the control rats. Iptakalim treatment did not affect food consumption in either the normal chow-fed or fructose-fed rats, but rosiglitazone treatment significantly increased food intake in the fructosefed rats. Iptakalim treatment did not affect body weight or food consumption in either the normal chow-fed or fructosefed rats (data not shown). IR associated with hypertension in the FFRs was characterized by an elevated SBP, decreased GIR, and insulin sensitive index (ISI) with diabetic manifestations of increased fasting blood levels of insulin (FBI) and glucose (FBG). Treatment with iptakalim at a dose of 1, 3, or 9 $\mathrm{mg} \cdot \mathrm{kg}^{-1} \cdot \mathrm{d}^{-1}$ or the PPARY agonist rosiglitazone at $5 \mathrm{mg} \cdot \mathrm{kg}^{-1} \cdot \mathrm{d}^{-1}$ for 4 weeks prevented IR associated with hypertension and hyperinsulinemia, and the SBP (Figure 4A), FBI (Figure 4B), 

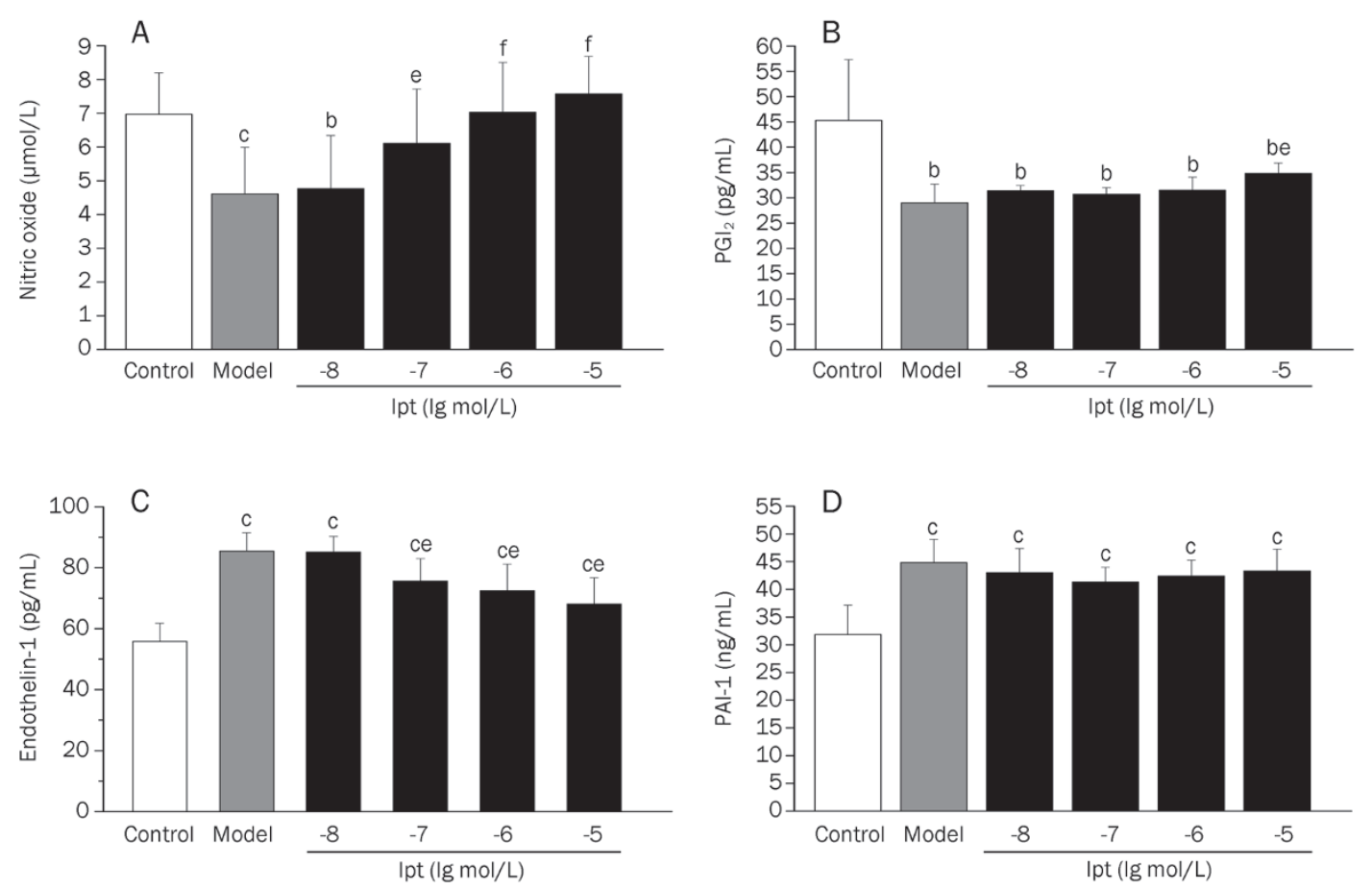

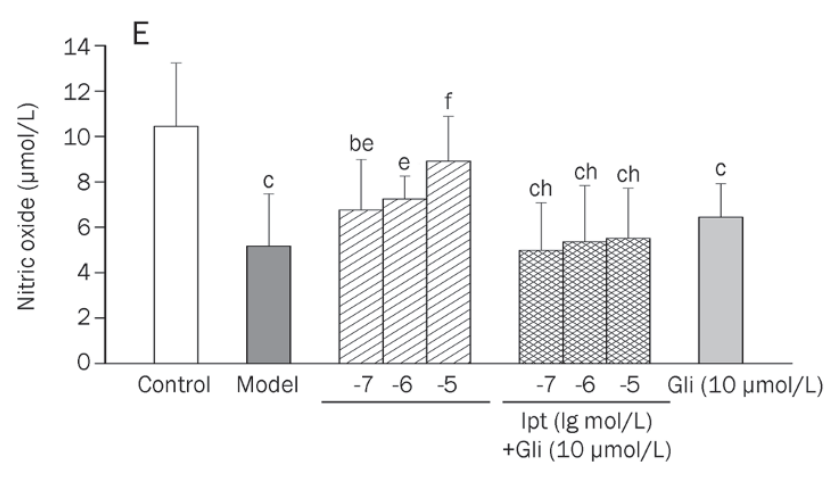

FBG (Figure 4C), GIR (Figure 4D), and ISI (Figure 4E) were normalized. In addition, iptakalim had no effect on the above parameters in the control rats fed a normal diet.

\section{Activation of $\mathrm{K}_{\text {ATP }}$ ameliorates fructose-induced endothelial} dysfunction in vivo

Endothelial dysfunction was characterized by impaired vascular relaxation induced by acetylcholine in aorta from the FFRs. The endothelium-dependent vascular relaxation induced by acetylcholine at concentrations of $0.1,1,10$, or $100 \mu \mathrm{mol} /$ $\mathrm{L}$ was significantly decreased in the in vitro aortic preparations isolated from the FFRs. This change was significantly improved by treatment with iptakalim at the doses of 1,3 , or 9 $\mathrm{mg} \cdot \mathrm{kg}^{-1} \cdot \mathrm{d}^{-1}$ for 4 weeks or by treatment with rosiglitazone (Figure 5). Serum levels of NO (Figure 6A) decreased, whereas ET-1 (Figure 6B) increased in the FFRs, which was improved by treatment with iptakalim at a dose of $3 \mathrm{mg} \cdot \mathrm{kg}^{-1} \cdot \mathrm{d}^{-1}$ for 4 weeks. These findings suggest that endothelial dysfunction can be restored by treatment with iptakalim in vivo.

In addition, serum levels of angiotensin II increased from
Figure 1. Pretreatment with iptakalim improves IR-induced endothelial dysfunction in vitro. The protective effect of iptakalim (Ipt) on endothelial dysfunction induced by IR with hyperinsulinemia was investigated in vitro. HUVECs were preincubated with Ipt for $6 \mathrm{~h}$ and then incubated with wortmannin (a PI-3 kinase inhibitor, $50 \mathrm{nmol} / \mathrm{L}$ ) and insulin (100 nmol/L) for $20 \mathrm{~h}$. The effects of Ipt on the production of nitric oxide $(A), \mathrm{PGI}_{2}(\mathrm{~B})$, endothelin-1 (C), and PAI-1 (D) were evaluated. The increased NO release by Ipt was inhibited by glibenclamide (Gli). Gli $\left(10^{-5} \mathrm{~mol} / \mathrm{L}\right)$ was added 1 $\mathrm{h}$ before Ipt pretreatment (E). The data are expressed as the mean $\pm S D$ ( $n=8$ separate sets of experiments). ${ }^{b} P<0.05,{ }^{\circ} P<0.01$ vs control group; ${ }^{\mathrm{e}} P<0.05,{ }^{\mathrm{f}} P<0.01$ vs model group, ${ }^{\mathrm{h}} P<0.05$ vs the data obtained in the absence of Gli.

$597.63 \pm 55.97(n=10)$ to $796.48 \pm 113.62(n=10) \mathrm{ng} / \mathrm{mL}(P<0.01$ vs normal control group) in the FFRs. However, these increased levels of angiotensin II were not influenced by treatment with iptakalim at a dose of $3 \mathrm{mg} / \mathrm{kg}$ po qd for 4 weeks, and the serum content of angiotensin II remained high at $808.30 \pm 87.03$ $\mathrm{ng} / \mathrm{mL}$ ( $n=10, P>0.05$ vs model group). However, the levels of angiotensin II decreased to $589.78 \pm 67.96(10) \mathrm{ng} / \mathrm{mL}(P<0.01$ vs model group; $P>0.05$ vs normal control group) with treatment with enalapril at a dose of $3 \mathrm{mg} \cdot \mathrm{kg}^{-1} \cdot \mathrm{d}^{-1}$ (Figure 6C). These results are consistent with those observed in hypertensive renal damage and renal injury induced by hyperuricemia ${ }^{[25,26]}$. Because iptakalim has no effect on the renin-angiotensin system, its benefit on IR associated with hypertension is entirely different from those of drugs acting on the RAS.

\section{Discussion}

Essential hypertension and insulin resistance are inextricably linked ${ }^{[1]}$. Approximately 50 percent of patients with hypertension can be considered to have insulin resistance and hyperinsulinemia ${ }^{[27]}$. Studies in both hypertensive humans and rodent 

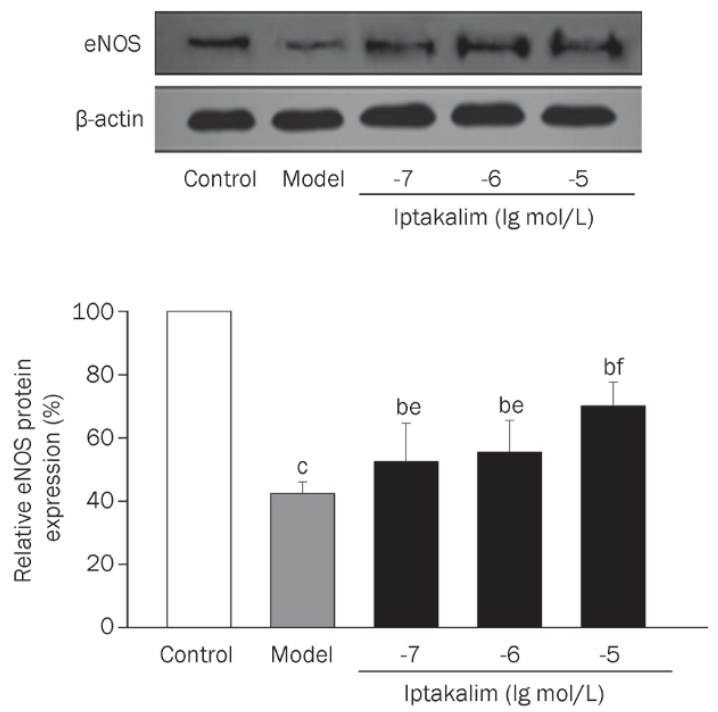

Figure 2. Effect of iptakalim on eNOS protein expression in HUVECs. Western blot analysis of eNOS protein expression in endothelial cells was performed with a rabbit monoclonal anti-eNOS antibody. An equal amount of protein was loaded in each lane. The density of the bands was quantified by densitometric scanning, and the relative density was calculated by setting the density of the control band as $100 \%$. The data are expressed as the mean \pm SD ( $n=3$ separate sets of experiments). ${ }^{\mathrm{b}} P<0.05,{ }^{\mathrm{c}} P<0.01$ compared to the control group. ${ }^{\mathrm{e}} P<0.05,{ }^{\mathrm{f}} P<0.01$ compared to the model group.

models of hypertension suggest that IR and/or compensatory hyperinsulinemia have an important role in blood pressure regulation and may predispose individuals to develop hypertension. However, an antihypertensive drug that reduces $\mathrm{BP}$ does not necessarily improve IR. Of the current first-line antihypertensive agents, $\beta$-adrenergic receptor blockers and diuretics can impair insulin sensitivity, and the metabolic effects of calcium channel blockers are still controversial ${ }^{[28]}$. Only angiotensin-converting enzyme (ACE) inhibitors and angiotensin II (Ang) II type 1 (AT1) receptor antagonists have been reported to be beneficial in patients with $\mathrm{IR}^{[7]}$. Drugs that can lower blood pressure and reverse the associated IR are still lacking, which reveals the need for developing new drugs that can reduce blood pressure and improve IR.

Recent studies have demonstrated that endothelial dysfunction is likely to be one of the most important underlying pathophysiological mechanisms that might serve as the link between IR and hypertension. The endothelium releases many vasoactive mediators that regulate cardiovascular function and, importantly, contribute to maintaining homeostasis. Endothelial dysfunction has been attributed to various cardiovascular risk factors and is mainly characterized by an imbalance between NO and ET-1 signaling, which contributes to further progression of hypertension. Insulin resistance is characterized by pathway-specific impairment in PI3-kinasedependent signaling, which in the endothelium, may also cause an imbalance between NO and endothelin-1, leading to decreased blood flow and worsening insulin resistance. Ther- apeutic interventions in animal models and human studies have indicated that improving endothelial dysfunction ameliorates insulin resistance, whereas improving insulin sensitivity ameliorates endothelial dysfunction ${ }^{[11]}$. Thus, if an antihypertensive agent protects against endothelial dysfunction, it is very likely to improve IR in hypertensive individuals.

Iptakalim is a novel $\mathrm{K}_{\text {ATP }}$ channel opener with potent antihypertensive properties, which have been confirmed in phase 3 clinical trials in China, and has selective activation of the SUR2B/Kir6.1 channel, resulting in the protection of endothelial function ${ }^{[14-18]}$. Iptakalim has protective effects against endothelial dysfunction induced by low-density lipoprotein, homocysteine, hyperglycemia, and hypertension ${ }^{[16]}$. In this study, we demonstrated for the first time that iptakalim improves IR associated with hypertension by restoring the balance between NO and ET-1 signaling in the endothelium.

First, we performed experiments to mimic IR-induced endothelial dysfunction. Simultaneous treatment of endothelial cells with wortmannin and high insulin levels blunts the PI3-kinase-dependent effects of insulin, such as the induction of eNOS expression and the release of NO, and increases the production of ET-1, which is consistent with previous reports ${ }^{[20,24]}$. Iptakalim rebalanced the release of NO and the production of ET-1 but had little effects on $\mathrm{PGI}_{2}$ and PAI-1. The effect of iptakalim on NO release was abolished by glibenclamide, a specific blocker of $\mathrm{K}_{\mathrm{ATP}}$ channels. Furthermore, iptakalim was able to increase eNOS protein expression in endothelial cells. These observations confirm previous results indicating that iptakalim potently protects against endothelial dysfunction $^{[16-19]}$. Iptakalim can improve IR-induced endothelial dysfunction and ameliorate the impaired insulin-PI3Kinase-NO signaling pathway via opening the SUR2B/Kir6.1 subtype of $\mathrm{K}_{\text {ATP }}$ channel. Thus, we hypothesized that iptakalim could ameliorate IR in vivo through endothelial protection induced by activating the SUR2B/Kir6.1 channel.

Several animal models have been used to study the relationship between IR and the development of hypertension, including SHRs and FFRs ${ }^{[29-32]}$. The SHR is a widely used genetic model of essential hypertension and the associated metabolic disturbances, including IR and endothelial dysfunction. Recent advances in the genetics of the SHR have revealed that there are some specific genes that influence blood pressure and $\mathrm{IR}^{[33]}$. The FFR is another useful model of acquired systolic hypertension that displays numerous features of the metabolic syndrome including IR. Rats fed a high fructose diet exhibit IR/hyperinsulinemia and hypertension, which is independent of genetic contributions ${ }^{[34]}$. In our study, these two different animal models were used to identify the effect of iptakalim on IR. Because of the effects of ACEIs and y peroxisome proliferator-activated receptors (PPARY) agonists on insulin-resistance states have been well documented ${ }^{[35]}$, we chose different ACEIs (benazepril for the SHRs and enalapril for the FFRs), and a PPARY agonist (rosiglitazone for the FFRs) as positive controls to demonstrate the reliability of the rat models of IR associated with hypertension.

Although growing evidence demonstrates that several 
A

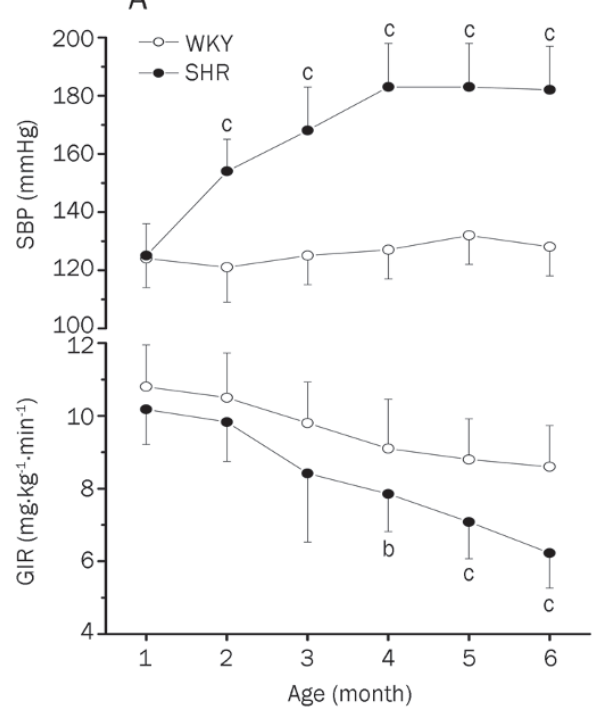

C
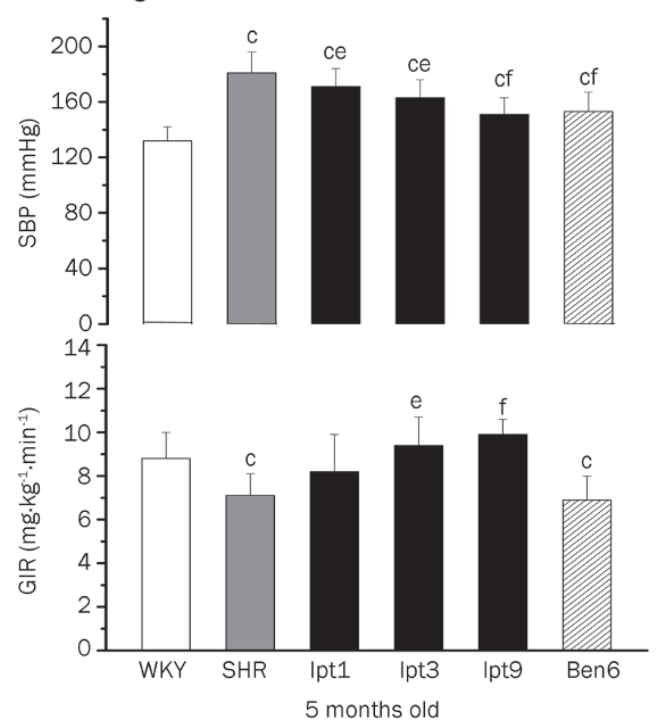

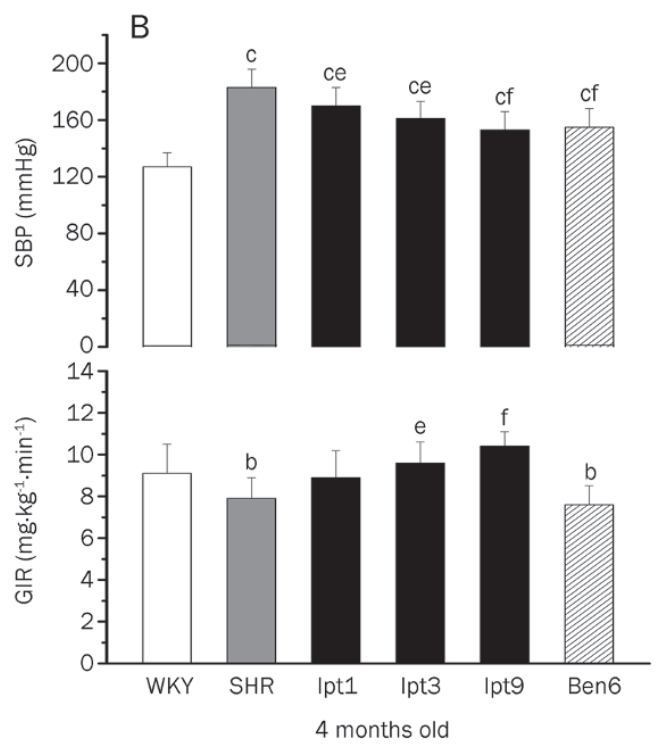

D
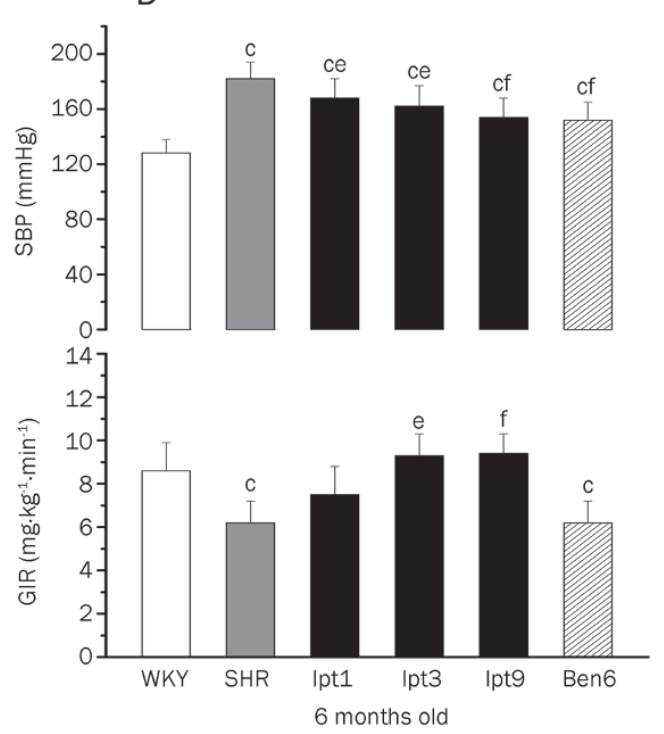

Figure 3. Treatment with iptakalim improves insulin resistance (IR) associated with hypertension in spontaneously hypertensive rats (SHRs). (A) The changes in the systolic blood pressure (SBP) and glucose infusion rate (GIR) in the SHRs and in the age-matched WKY rats at 1, 2, 3, 4, 5, and 6 months of age. (B, C, D) Eight-week treatment with iptakalim reduced the SBP and attenuated IR in SHRs. The daily therapy with vehicle, iptakalim 1 , 3 , or $9 \mathrm{mg} \cdot \mathrm{kg}^{-1} \cdot \mathrm{d}^{-1}$ (Ipt1, Ipt3, Ipt9), or benazepril $6 \mathrm{mg} \cdot \mathrm{kg}^{-1} \cdot \mathrm{d}^{-1}$ (Ben 6) for 8 weeks was performed before (SHRs 2 (B) and 3 (C) months of age) and after (SHRs 4 (D) months of age) IR was established. When the treatments were completed, these SHRs were 4 (B), 5 (C), or 6 (D) months old. The data are expressed as the mean \pm SD $(n=6$ per group). ${ }^{\mathrm{b}} P<0.05,{ }^{\mathrm{c}} P<0.01 \mathrm{vs}$ age-matched WKY rats, ${ }^{e} P<0.05$, ${ }^{\mathrm{f}} P<0.01$ vs age-matched SHRs. related mechanisms for IR contribute to the pathogenesis of hypertension in the SHRs, the exact cause of this disorder remains unclear. In addition, whether IR is a "reason" or a "result" for the progression of hypertension is yet to be elucidated. Our results indicate that hypertension develops before IR. Furthermore, we have shown for the first time that the GIR was improved after 8 weeks of iptakalim treatment in SHRs. There are at least two reasons that may explain this phenomenon. First, the lower blood pressure may result from iptakalim directly. Another possibility is that the lower blood pressure results from the restoration of endothelial function by iptakalim. Therefore, it is very important to further explore the molecular mechanism by which iptakalim affects IR associated with hypertension in SHRs.

A substantial number of studies suggest a relationship among the occurrence of IR, endothelial dysfunction and hypertension in FFRs, but the cause and effect among them has yet to be elucidated. Katakam and colleagues reported that hyperinsulinemia occurs following $3 \mathrm{~d}$ of high fructose feeding, IR develops after 7-10 d, endothelial dysfunction was observed following $18 \mathrm{~d}$ and hypertension was found after 4 weeks $^{[33,36,37]}$. Based on the order in which they develop, we designed this study such that the iptakalim treatments were performed after hypertension had developed after 4 weeks of the diet. We found that treating the FFRs with iptakalim for 4 weeks resulted in lower blood pressures and restored the balance between the production of NO and secretion of ET-1. Furthermore, the impaired ACh-induced endothelium-dependent relaxation of aortic tissue derived from the FFRs was improved significantly after 4 weeks of iptakalim administration. In agreement with other reports ${ }^{[31,38]}$, we have demonstrated that iptakalim has a direct endothelial effect. Thus, the similar in vivo and in vitro effects of iptakalim indicate that the positive effects of iptakalim on vascular function are mainly 
A

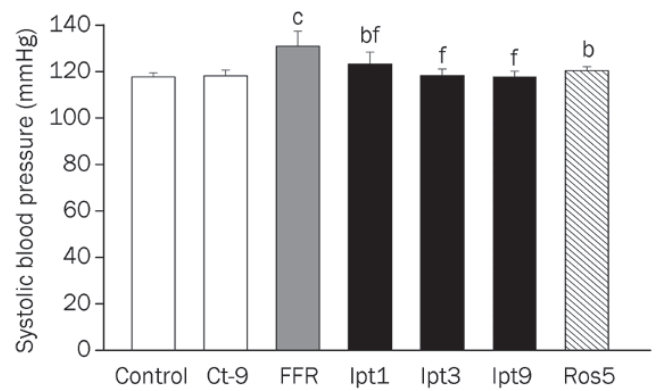

C

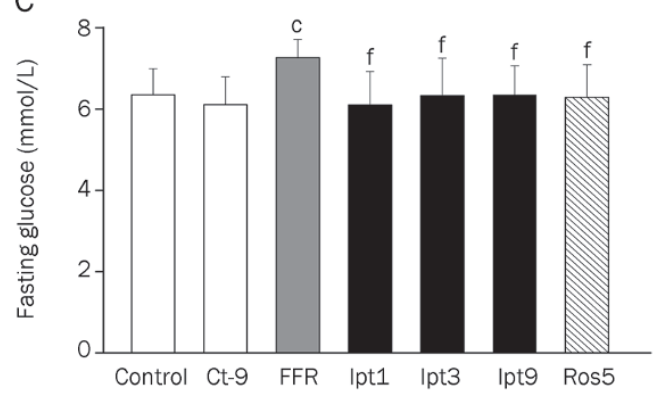

E

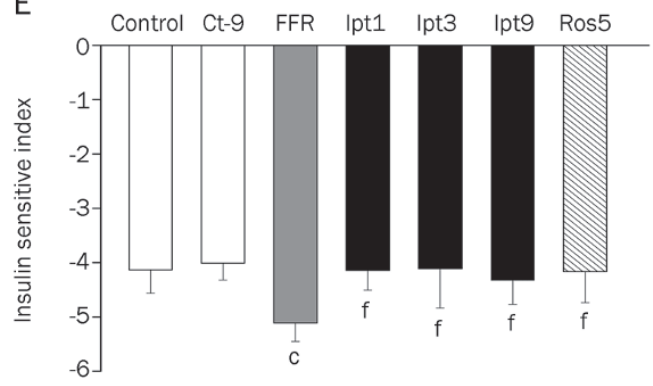

B

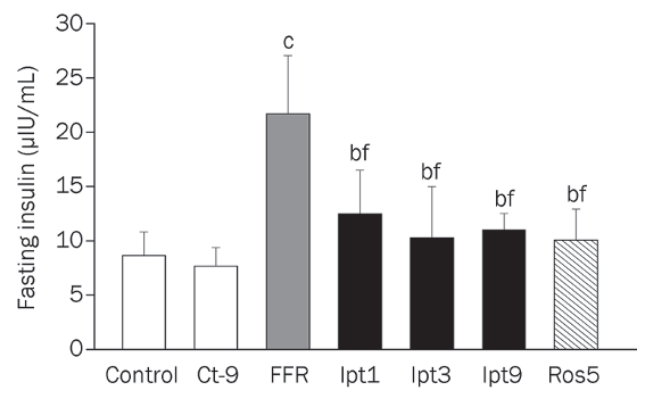

D

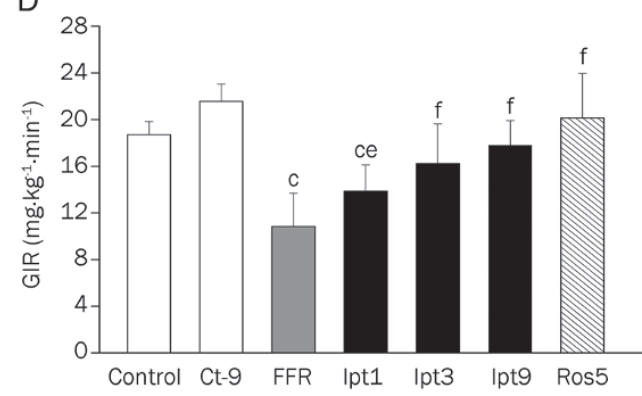

Figure 4. Treatment with iptakalim improved insulin resistance (IR) associated with hypertension in fructose-fed rats (FFR). In Sprague-Dawley rats fed either a normal (Ctrl) or high fructose-fed (FFR) diet for 8 weeks, iptakalim and rosiglitazone were administered orally at 1,3 , or $9 \mathrm{mg} \cdot \mathrm{kg}^{-1} \cdot \mathrm{d}^{-1}$ (Ipt1, Ipt3, Ipt9) and $5 \mathrm{mg} \cdot \mathrm{kg}^{-1} \cdot \mathrm{d}^{-1}$ (Ros 5 ), respectively, for the last 4 weeks in the high-fructose diet group. Iptakalim was administered orally at $9 \mathrm{mg} \cdot \mathrm{kg}^{-1} \cdot \mathrm{d}^{-1}(\mathrm{Ct}-9)$ for the last 4 weeks in the control diet group. At the end of 8 weeks, the SBP (A) was measured using the tail cuff method. Fasting blood samples were obtained to measure insulin (B) and glucose (C). Insulin sensitivity was assessed by the GIR (D) and ISI (E). The data are expressed as the mean \pm SD ( $n=14$ per group). ${ }^{b} P<0.05,{ }^{C} P<0.01$ vs control group. ${ }^{e} P<0.05,{ }^{f} P<0.01$ vs model group.

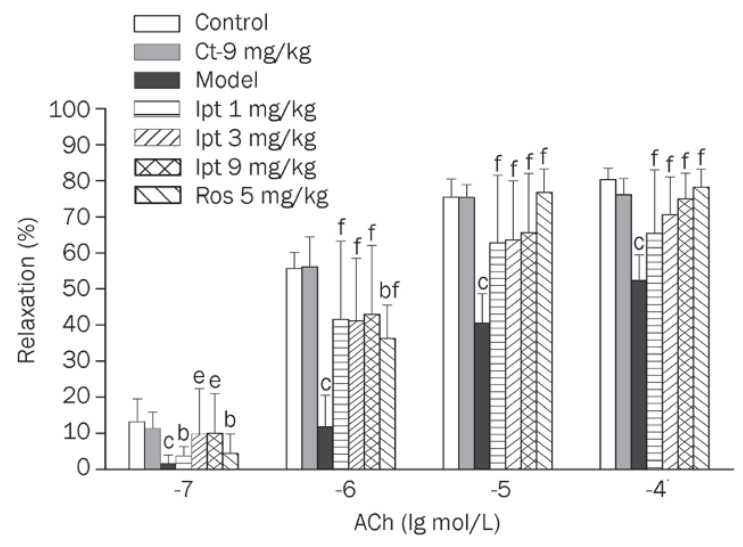

Figure 5. Treatment with iptakalim or rosiglitazone improved acetylcholineinduced endothelium-dependent vascular relaxation in the isolated aortic rings derived from the FFRs. Normal rats (Control), iptakalim control (Ct$9 \mathrm{mg} / \mathrm{kg}$ ), FFRs (Model), and the FFRs treated with iptakalim (Ipt 1, 3, or $9 \mathrm{mg} / \mathrm{kg}$ ) and rosiglitazone (Ros-5 mg/kg) for 4 weeks. The data are expressed as the mean \pm SD ( $n=6$ per group). ${ }^{\mathrm{b}} P<0.05,{ }^{\mathrm{C}} P<0.01$ vs control group. ${ }^{\mathrm{e}} P<0.05,{ }^{\mathrm{f}} P<0.01$ vs model group. due to its direct effects on endothelial function.

Interestingly, in this study, we have demonstrated that iptakalim treatment improves insulin sensitivity, normalizes the GIR, and decreases plasma insulin levels in FFRs. Because therapeutic interventions in animal models and human studies have confirmed that improving endothelial dysfunction ameliorates insulin resistance ${ }^{[11]}$, it is reasonable to postulate that improvements in IR by iptakalim are due to its amelioration of endothelial dysfunction.

It is well known that ACE inhibitors may directly improve endothelial dysfunction by blocking local endothelial angiotensin II production. Our results showed that enalapril can inhibit the increased serum concentration of Ang II, while iptakalim has no direct effect on this change in the FFRs, which suggests that the mechanism for iptakalim-induced improvement in IR differs from that of enalapril and occurs through a novel molecular pathway.

Based upon the above investigation, it appears reasonable to conclude that iptakalim, a $\mathrm{K}_{\mathrm{ATP}}$ channel opener, protects against IR-induced endothelial dysfunction by restoring the 

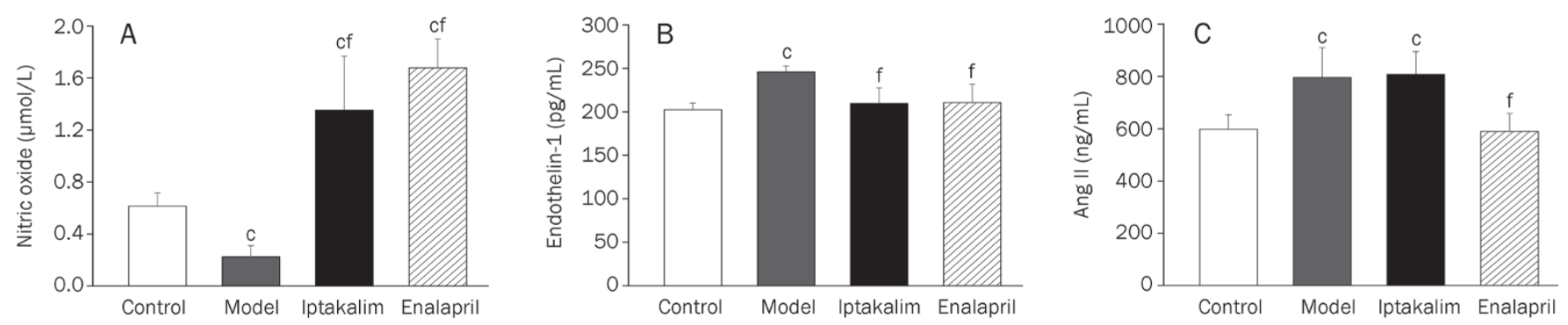

Figure 6. Iptakalim ameliorates endothelial dysfunction in FFRs. Treatment with iptakalim or enalapril $3 \mathrm{mg} \cdot \mathrm{kg}^{-1} \cdot \mathrm{d}^{-1}$ for 4 weeks increased serum nitric oxide levels and decreased serum endothelin-1 levels in FFRs (A, B). Iptakalim treatment had no effect on the serum angiotensin II (Ang II) level, which was different compared to enalapril (C). The data are expressed as the mean $\pm \mathrm{SD}(n=8)$. ${ }^{\mathrm{C}} P<0.01 \mathrm{vs}$ control group; ${ }^{\mathrm{f}} P<0.01 \mathrm{vs}$ model group.

balance between NO and ET-1 signaling, and ameliorates the IR associated with hypertension.

\section{Conclusions}

Iptakalim can protect endothelial cells by activating $\mathrm{K}_{\mathrm{ATP}}$ through the preferential activation of the SUR2B/Kir6.1 subtypes of $K_{\text {ATP }}$ expressed in the endothelium. In this study, we first investigated the effects of iptakalim on IR in SHRs and FFRs and then explored the molecular mechanism by which it protects against endothelial dysfunction in vivo and in vitro. These findings suggest that iptakalim improves the IR associated with hypertension through restoring the balance between $\mathrm{NO}$ and ET-1 signaling and that targeting the SUR2B/Kir6.1 subtype of $\mathrm{K}_{\mathrm{ATP}}$ is a new strategy for ameliorating the IR associated with hypertension.

\section{Acknowledgements}

This study was supported by grants from the National New Drug Research and Development Key Project (No 2008ZX09101-006, 2008ZXJ09004-018 and 2009ZX09301-002), the State Key Basic Research and Development from the Ministry of Science and Technology of China (№ GT1998051112), the 863-High Technology Research and Development Program Plan (No 2002AA2Z3137), the National 1035 Project (No 969010101) of China and the New Drug Development of Beijing Key Project (№ D0204003040721).

\section{Author contribution}

Hai WANG and Guo-shu LIU designed the research. Yu WANG, Fu-hu ZENG, Zhi-yuan PAN, Wen-yu CUI, and Ruhuan WANG performed the research. Yu WANG and Fu-hu ZENG analyzed the data. Yu WANG, Chao-liang LONG, and Zhi-yuan PAN wrote the paper.

\section{Abbreviations}

$\mathrm{K}_{\text {ATP }}$, ATP-sensitive potassium channel; IR, insulin resistance; SHR, spontaneously hypertensive rat; FFR, fructose-fed rat; GIR, glucose infusion rate; SBP, systolic blood pressure; HUVEC, human umbilical vein endothelial cells; NO, nitric oxide; ET-1, endothelin-1; Ang II, angiotensin II; ACEI, angiotensin-converting enzyme inhibitor; 6-Keto-PGF Id $_{1}$, 6-Ketoprostaglandin- $\mathrm{F}_{1 \alpha}$; PAI-1, plasminogen activator inhibitor-1; PPARY, $\mathrm{Y}$ peroxisome proliferator-activated receptors.

\section{References}

1 Ferrannini E, Buzzigoli G, Bonadonna R, Giorico MA, Oleggini M, Graziadei L, et al. Insulin resistance in essential hypertension. N Engl J Med 1987; 317: 350-7.

2 Reddy KJ, Singh M, Bangit JR, Batsell RR. The role of insulin resistance in the pathogenesis of atherosclerotic cardiovascular disease: an updated review. J Cardiovasc Med (Hagerstown) 2010; 11: 633-47.

3 Penesova A, Cizmarova E, Belan V, Blazicek P, Imrich R, Vlcek M, et al. Insulin resistance in young, lean male subjects with essential hypertension. J Hum Hypertens 2011; 25: 391-400.

4 Perticone F, Sciacqua A, Maio R, Perticone M, Galiano Leone G, Bruni $\mathrm{R}$, et al. Endothelial dysfunction, ADMA and insulin resistance in essential hypertension. Int J Cardiol 2010; 142: 236-41.

5 Higashiura K, Ura N, Takada T, Li Y, Torii T, Togashi N, et al. The effects of an angiotensin-converting enzyme inhibitor and an angiotensin II receptor antagonist on insulin resistance in fructose-fed rats. Am J Hypertens 2000; 13: 290-7.

6 Umeda M, Kanda T, Murakami M. Effects of angiotensin II receptor antagonists on insulin resistance syndrome and leptin in sucrose-fed spontaneously hypertensive rats. Hypertens Res 2003; 26: 485-92.

7 Pahor M, Psaty BM, Furberg CD. Treatment of hypertensive patients with diabetes. Lancet 1998; 32: s18-23.

8 Duncan ER, Crossey PA, Walker S, Anilkumar N, Poston L, Douglas G, et al. Effect of endothelium-specific insulin resistance on endothelial function in vivo. Diabetes 2008; 57: 3307-14.

9 Katakam PV, Ujhelyi MR, Hoenig ME, Miller AW. Endothelial dysfunction precedes hypertension in diet-induced insulin resistance. Am J Physiol 1998; 275: R788-92.

10 Tziomalos K, Athyros VG, Karagiannis A, Mikhailidis DP. Endothelial dysfunction in metabolic syndrome: prevalence, pathogenesis and management. Nutr Metab Cardiovasc Dis 2010; 20: 140-6.

11 Kim JA, Montagnani M, Koh KK, Quon MJ. Reciprocal relationships between insulin resistance and endothelial dysfunction: molecular and pathophysiological mechanisms. Circulation 2006; 113: 1888904.

12 Potenza MA, Marasciulo FL, Chieppa DM, Brigiani GS, Formoso G, Quon MJ, et al. Insulin resistance in spontaneously hypertensive rats is associated with endothelial dysfunction characterized by imbanlace between NO and ET-1 production. Am J Physiol 2005; 289: H813-22.

13 Minamino T, Hori M. Protecting endothelial function: a novel therapeutic target of ATP-sensitive potassium channel openers. Cardiovasc Res 2007; 73: 448-9.

14 Pan Z, Huang J, Cui W, Long C, Zhang Y, Wang H. Targeting hypertension with a new ATP sensitive potassium channel opener iptakalim. 
J Cardiovasc Pharmacol 2010; 56: 215-28.

15 Duan RF, Cui WY, Wang H. Association of the antihypertensive response of iptakalim with KCNJ11 (Kir6.2 gene) polymorphisms in Chinese Han hypertensive patients. Acta Pharmacol Sin 2011; 32 : 1078-84.

16 Wang $\mathrm{H}$, Long $\mathrm{C}$, Duan Z, Shi C, Jia G, Zhang Y. A new ATP-sensitive potassium channel opener protects endothelial function in cultured aortic endothelial cells. Cardiovasc Res 2007; 73: 497-503.

17 Gao S, Long $\mathrm{CL}$, Wang $\mathrm{RH}$, Wang $\mathrm{H}$. $\mathrm{K}_{\text {ATP }}$ activation prevents progression of cardiac hypertrophy to failure induced by pressure overload via protecting endothelial function. Cardiovasc Res 2009; 83: 444-56.

18 Tang $\mathrm{Y}$, Long $\mathrm{CL}$, Wang RH, Cui W, Wang $\mathrm{H}$. Activation of SUR2B/ Kir6.1 subtype of adenosine triphosphate-sensitive potassium channel improves pressure overload induced cardiac remodeling via protecting endothelial function. J Cardiovasc Pharmacol 2010; 56: 345-53.

19 Zhao RJ, Wang H. Chemerin/ChemR23 signaling axis is involved in the endothelial protection by $\mathrm{K}_{\text {ATP }}$ channel opener iptakalim. Acta Pharmacol Sin 2011; 32: 573-80.

20 Montagnani M, Golovchenko I, Kim I, Koh GY, Goalstone ML, Mundhekar AN, et al. Inhibition of phosphatidylinositol 3-kinase enhances mitogenic actions of insulin in endothelial cells. J Biol Chem 2002; 277: 1794-9.

21 Vaziri ND, Wang XQ. cGMP-mediated negative-feedback regulation of endothelial nitric oxide synthase expression by nitric oxide. Hypertension 1999; 34: 1237-41.

22 Furukawa LN, Kushiro T, Asagami T, Takahashi A, Kanmatsuse K, Ishikawa K. Variations in insulin sensitivity in spontaneously hypertensive rats from different sources. Metabolism 1998; 47: 493-6.

23 Katakam PV, Ujhelyi MR, Hoenig M, Miller AW. Metformin improves vascular function in insulin-resistant rats. Hypertension 2000; 35 : 108-12.

24 Li R, Zhang H, Wang W, Wang X, Huang Y, Huang C, et al. Vascular insulin resistance in prehypertensive rats: role of PI3-kinase/Akt/ eNOS signaling. Eur J Pharmacol 2010; 628: 140-7.

25 Xue H, Zhang YL, Liu GS, Wang H. A new ATP-sensitive potassium channel opener protects the kidney from hypertensive damage in experimental hypertension. J Pharmacol Exp Ther 2005; 315: 501-9.
26 Long CL, Qin XC, Pan ZY, Chen K, Zhang YF, Cui WY, et al. Activation of ATP-sensitive potassium channels protects vascular endothelial cells from hypertension and renal injury induced by hyperuricemia. J Hyperten 2008; 26: 2326-38.

27 Pollare $\mathrm{T}$, Lithell $\mathrm{H}$, Berne $\mathrm{C}$. Insulin resistance is a characteristic feature of primary hypertension in dependent of obesity. Metabolism 1990; 39: 167-74.

28 Oron-Herman M, Sela BA, Rosenthal T. Risk reduction therapy for syndrome X: comparison of several treatments. Am J Hypertens 2005; 18: 372-8.

29 Modon CE, Reaven GM. Evidence of abnormalities of insulin metabolism in rats with spontaneously hypertension. J Metabolism 1988; 37: 303-5.

30 Verma S, Yao L, Stewart DJ, Dumont AS, Anderson TJ, McNeill JH. Endothelin antagonism uncovers insulin-mediated vasorelaxation in vitro and in vivo. Hypertension 2001; 37: 328-33.

31 Misurski DA, Wu SQ, McNeill JR, Wilson TW, Gopalakrishnan V. Insulin-induced biphasic response in rat mesenteric vascular bed: role of endothelin. Hypertension 2001; 37: 1298-302.

32 Verma S, Bhanot S, McNeill JH. Effects of chronic endothelin blockade in hyperinsulinemia hypertensive rats. Am J Physiol 1995; 269: H2017-21.

33 Kurtz TW. Recent advances in genetics of the spontaneously hypertensive rat. Curr Hypertens Rep 2010; 12: 5-9.

34 Tran LT, Yuen VG, McNeill JH. The fructose-fed rats: a review on the mechanism of fructose-induced insulin resistance and hypertension. Mol Cell Biochem 2009; 332: 145-59.

35 Potenza MA, Marasciulo FL, Tarquinio M, Quon MJ, Montagnani M. Treatment of spontaneously hypertensive rats with rosiglitazone and/ or enalapril restores balance between vasodilator and vasoconstrictor actions of insulin with simultaneous improvement in hypertension and insulin resistance. Diabetes 2006; 55: 3594-603.

36 Katakam PV, Ujhelyi MR, Hoenig ME, Miller AW. Endothelial dysfunction precedes hypertension in diet-induced insulin resistance. Am J Physiol 1998; 275: R788-92.

37 Hwang IS, Ho H, Hoffman BB, Reaven GM. Fructose-induced insulin resistance and hypertension in rats. Hypertension 1987; 10: 512-6.

38 Wilkes JJ, Hevener A, Olefsky J. Chronic endothelin-1 treatment leads to insulin resistance in vivo. Diabetes 2003; 52: 1904-9. 